Document downloaded from:

http://hdl.handle.net/10251/87294

This paper must be cited as:

Guterman, A.; Herrero Debón, A.; Thome, N. (2016). New matrix partial order based spectrally orthogonal matrix decomposition. Linear and Multilinear Algebra. 64(3):362-374. doi:10.1080/03081087.2015.1041365

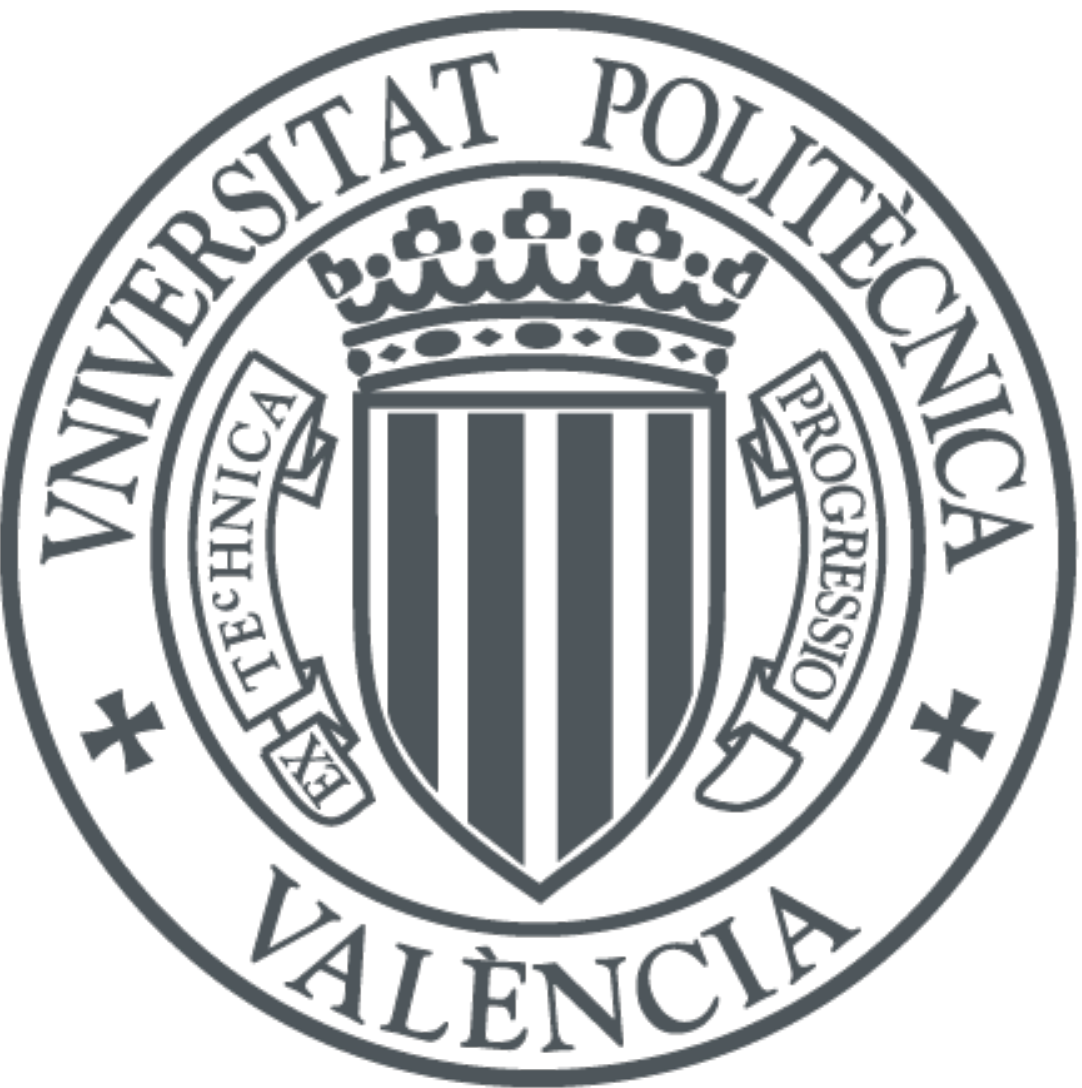

The final publication is available at

http://dx.doi.org/10.1080/03081087.2015.1041365

Copyright Taylor \& Francis

Additional Information 


\section{New matrix partial order based on spectrally orthogonal matrix decomposition}

Article in Linear and Multilinear Algebra · May 2015

DOI: $10.1080 / 03081087.2015 .1041365$

CITATIONS

2

3 authors:

\section{Alexander Guterman}

Lomonosov Moscow State University

96 PUBLICATIONS 625 CITATIONS

SEE PROFILE

Nestor Thome

Universitat Politècnica de València

75 PUBLICATIONS 413 CITATIONS

SEE PROFILE
READS

101
Alicia Herrero

Universitat Politècnica de València

31 PUBLICATIONS 97 CITATIONS

SEE PROFILE

Some of the authors of this publication are also working on these related projects:

Project

Generalizaed inverses View project

Project
Nonnegativity View project 


\title{
New matrix partial order based on spectrally orthogonal matrix decomposition
}

\author{
Alexander Guterman \\ Alicia Herrero \\ Néstor Thome
}

\begin{abstract}
We investigate partial orders on the set of complex square matrices and introduce a new order relation based on spectrally orthogonal matrix decompositions. We also establish the relation of this concept with the known orders.
\end{abstract}

Keywords. Spectrum, sharp order, minus order, spectrally orthogonal matrix decomposition

\section{Introduction}

The spectral theorem is a well-known technique mainly used in the literature for working with diagonalizable matrices. However, for general matrices (that is, diagonalizable or not) this tool (see [13, pp. 603]) has not been so much exploited in relation to partial orders. In this paper we apply matrix canonical forms defined in [4], namely spectrally orthogonal matrix decompositions, which are useful for general matrices. This gives us the possibility to introduce and investigate some new partial orders.

Let $\mathbb{C}$ be the field of complex numbers and $M_{n}(\mathbb{C})$ be the set of $n \times n$ matrices over $\mathbb{C}$. We denote by $I_{n}$ the identity matrix of size $n$ and by $O_{n}$ the $n \times n$ zero matrix. We omit the subscripts for $I_{n}$ and $O_{n}$ if their size is clear from the context. Let $E_{i j}$ be the matrix with 1 in $(i, j)$-th position and zeros elsewhere. Two matrices $A, B \in M_{n}(\mathbb{C})$ are said to be orthogonal, and denoted by $A \perp B$, if $A B=B A=O$. The symbols rk and Spec will stand for the rank and the spectrum of a square matrix, respectively.

A. Guterman: Department of Mathematics and Mechanics, Moscow State University, 119991, GSP-1, Moscow, Russia; e-mail: guterman@ list.ru

A. Herrero: Universitat Politècnica de València, Camino de Vera s/n, 46022, València, Spain; e-mail: aherrero@mat.upv.es

N. Thome: Universitat Politècnica de València, Camino de Vera s/n, 46022, València, Spain; e-mail: njthome@mat.upv.es

Mathematics Subject Classification (2010): Primary: 15A09, Secondary: 06A06 
We recall that a matrix $M \in M_{n}(\mathbb{C})$ has index $\ell\left(\operatorname{Ind} M=\ell\right.$ ) if $\operatorname{rk} M^{\ell}=\operatorname{rk} M^{\ell+1}$ and $\ell$ is the smallest nonnegative integer with this property. The core-nilpotent decomposition of an arbitrary matrix $M \in M_{n}(\mathbb{C})$ is given by the sum $M=C_{M}+N_{M}$, where $C_{M} \perp$ $N_{M}$, Ind $C_{M} \leq 1$, and $N_{M}=O$ or $N_{M}$ is a nilpotent matrix. By convention, if $M=O$, we set $C_{M}=N_{M}=O$. This decomposition always exists and is unique (see [3, Chapter 4.8]).

Let $M \in M_{n}(\mathbb{C})$. A matrix $X \in M_{n}(\mathbb{C})$ is called a $g$-inverse of $M$ if it satisfies $M X M=M$ and will be denoted by $X=M^{-}$. If $X$ is a $g$-inverse of $M$ that satisfies $X M X=X$ and $M X=X M$, it is called a group inverse of $M$ and is denoted by $X=M^{\#}$. If $X$ satisfies $X M X=X, M X=X M$, and $M^{\ell+1} X=M^{\ell}$, with Ind $M=\ell$, it is called a Drazin inverse of $M$ and is denoted by $X=M^{D}$. The Drazin inverse is unique and its existence is guaranteed for every square matrix [3]. In the particular case Ind $M \leq 1$, the Drazin inverse becomes the group inverse.

Some generalized inverse matrices provide a method to define partial orders (see [15] and references therein, $[1,2,8,9,10,11,12,17])$.

Definition 1.1. $[6,16]$ The minus partial order on $M_{n}(\mathbb{C})$ is defined by $A \leq B$ if and only if $A^{-} A=A^{-} B$ and $A A^{-}=B A^{-}$for some $g$-inverse $A^{-}$of $A$.

Definition 1.2. [14] Let $A, B \in M_{n}(\mathbb{C})$ of index less than or equal to 1 . It is said that $A$ is below $B$ under the sharp partial order, and denoted by $A \stackrel{\sharp}{\leq} B$, if and only if $A^{\sharp} A=A^{\sharp} B$ and $A A^{\sharp}=B A^{\sharp}$.

When the Drazin inverse is used, the corresponding binary relation $\left(A^{D} A=A^{D} B\right.$ and $A A^{D}=B A^{D}$ ) does not give a partial order but a pre-order. However, using the core-nilpotent decomposition of both $A$ and $B$, the following notion is recalled.

Definition 1.3. [7] Let $A, B \in M_{n}(\mathbb{C})$. It is said that $A$ is below $B$ under the cn-order, and denoted by $A \stackrel{\text { cn }}{\leq} B$, if and only if $C_{A} \stackrel{\sharp}{\leq} C_{B}$ and $N_{A} \stackrel{-}{\leq} N_{B}$.

Alternative ways to define the minus and the sharp orders are the following:

Lemma 1.4. $[14,16]$ Let $A, B \in M_{n}(\mathbb{C})$. The following statements hold:

(a) $A \overline{\leq} B$ if and only if $\mathrm{rk}(B-A)=\operatorname{rk} B-\operatorname{rk} A$.

(b) $A \stackrel{\sharp}{\leq} B$ if and only if $A^{2}=B A=A B$, for $A$ and $B$ being of index at most 1 .

This paper is organized as follows. In Section 2 we present the spectrally orthogonal matrix decompositions and introduce two new binary relations based on these decompositions, namely $\stackrel{1}{\leq}$ and $\stackrel{2,3}{\leq}$. We prove that both relations are partial orders on $M_{n}(\mathbb{C})$. In 
Section 3 , we establish that $\stackrel{1}{\leq}$ is equivalent to the cn-order, which gives a characterization for the latter via a spectrally orthogonal decomposition. Also this section contains various examples, among them, there is an example showing that $\stackrel{2,3}{\leq}$ indeed provides a new relation on $M_{n}(\mathbb{C})$, in particular, it is different from the cn-order and other known order relations. In Section 4, sets of matrices which are majorized by an idempotent are considered. Finally, we establish that the $\stackrel{2,3}{\leq}$-order on $M_{n}(\mathbb{C})$ is not $\mathcal{G}$-based for any $n>1$.

\section{Partial orders and spectrally orthogonal decomposi- tions}

The notion of spectrally orthogonal matrix decompositions was introduced in [4]. These decompositions are used in the present paper, so we recall below some basic definitions and properties.

We consider the counting function $k_{M}: \mathbb{C} \times \mathbb{N} \rightarrow \mathbb{N} \cup\{0\}$ defined by the following rule: for $\lambda \in \mathbb{C}$ and $r \in \mathbb{N}$ the value $k_{M}(\lambda, r)$ equals to the number of Jordan blocks of $M \in M_{n}(\mathbb{C})$ of size $r$, corresponding to the eigenvalue $\lambda$. If there are no Jordan blocks of $M$ with $\lambda$ of size $r$ then $k_{M}(\lambda, r)=0$.

Moreover, the function $K_{M}: \mathbb{C} \rightarrow \mathbb{N} \cup\{0\}$, given by $K_{M}(\lambda)=\sum_{r=1}^{\infty} k_{M}(\lambda, r)$, determines the total number of Jordan blocks of $M$, corresponding to the eigenvalue $\lambda$.

Observe that $\operatorname{Spec} M=\left\{\lambda \in \mathbb{C} \mid K_{M}(\lambda)>0\right\}$. Now we are ready to define the following matrix functions:

Definition 2.1. Let $M=C_{M}+N_{M}$ be the core-nilpotent decomposition of $M \in M_{n}(\mathbb{C})$. We define the maps $S_{M}^{i}: \mathbb{C} \rightarrow M_{n}(\mathbb{C}), i=1,2,3$, by

(i) $S_{M}^{1}(0)=N_{M}$ and for any $\lambda \neq 0$ the matrix $S_{M}^{1}(\lambda)=X_{\lambda}$ where $X_{\lambda}$ is the unique (by $[4$, Lemma 2.14]) matrix such that the following three conditions hold

a) $X_{\lambda}^{\sharp} X_{\lambda}=X_{\lambda}^{\sharp} M=M X_{\lambda}^{\sharp}$,

b) $K_{X_{\lambda}}(\lambda)=K_{M}(\lambda)$,

c) $K_{X_{\lambda}}(\mu)=0$ for all $\mu \in \mathbb{C} \backslash\{0, \lambda\}$.

(ii) $S_{M}^{2}(\lambda)=S_{M+I}^{1}(\lambda+1)-S_{M}^{1}(\lambda)$ for all $\lambda \in \mathbb{C}$.

(iii) $S_{M}^{3}(\lambda)=S_{M}^{1}(\lambda)-\lambda S_{M}^{2}(\lambda)$ for all $\lambda \in \mathbb{C}$.

In the sequel we need several properties of these decompositions proved in [4], see also [5]:

Theorem 2.2. [4, Remark 2.16, Theorem 2.17] Let $M \in M_{n}(\mathbb{C})$. 
(i) If $\lambda \notin \operatorname{Spec} M$ then $S_{M}^{i}(\lambda)=$ Ofor $i=1,2,3$.

(ii) $\operatorname{rk}\left(S_{M}^{2}(\lambda)\right)=\operatorname{deg}_{\chi_{M}}(z-\lambda)$ is the multiplicity of $\lambda$ in the characteristic polynomial $\chi_{M}$.

(iii) $S_{M}^{i}(\lambda) \perp S_{M}^{j}(\mu)$ for all $\lambda \neq \mu, i, j=1,2,3$.

(iv) $S_{P^{-1} M P}^{i}(\lambda)=P^{-1} S_{M}^{i}(\lambda) P$ for all $\lambda \in \mathbb{C}, M \in M_{n}(\mathbb{C}), P \in G L_{n}(\mathbb{C})$, and $i=$ $1,2,3$.

(v) The matrix $S_{M}^{2}(\lambda)$ is idempotent for all $\lambda \in \mathbb{C}$.

(vi) The matrix $S_{M}^{3}(\lambda)$ is nilpotent or null for all $\lambda \in \mathbb{C}$.

(vii) $M=\sum_{\lambda \in \mathbb{C}} S_{M}^{1}(\lambda)=\sum_{\lambda \in \mathbb{C}}\left(\lambda S_{M}^{2}(\lambda)+S_{M}^{3}(\lambda)\right), I=\sum_{\lambda \in \mathbb{C}} S_{M}^{2}(\lambda)$.

These matrix functions and their properties allow us to give the following definition.

Definition 2.3. The decompositions $M=\sum_{\lambda \in \mathbb{C}} S_{M}^{1}(\lambda)$ and $M=\sum_{\lambda \in \mathbb{C}}\left(\lambda S_{M}^{2}(\lambda)+S_{M}^{3}(\lambda)\right)$ stated in Theorem 2.2 are called the spectrally orthogonal matrix decompositions of $M \in$ $M_{n}(\mathbb{C})$.

The following property of the matrix $S_{M}^{2}(\lambda)$ is very important.

Lemma 2.4. Let $M \in M_{n}(\mathbb{C})$. Then for $\lambda \in \mathbb{C}$ it holds that $\lambda \in \operatorname{Spec}(M)$ if and only if $S_{M}^{2}(\lambda) \neq O$.

Proof. Let $S_{M}^{2}(\lambda) \neq O$. Hence, by item (ii) of Theorem 2.2, $\operatorname{deg}_{\chi_{M}}(z-\lambda)>0$. Then $\lambda \in \operatorname{Spec}(M)$. The converse follows similarly.

From now on, we introduce and investigate the following binary relations on matrices based on spectrally orthogonal matrix decompositions.

Definition 2.5. For $A, B \in M_{n}(\mathbb{C})$ it is said that $A \stackrel{1}{\leq} B$ if $S_{A}^{1}(0) \stackrel{-}{\leq} S_{B}^{1}(0)$ and $S_{A}^{1}(\lambda) \stackrel{\sharp}{\leq}$ $S_{B}^{1}(\lambda)$ for all $\lambda \in \mathbb{C} \backslash\{0\}$.

Definition 2.6. For $A, B \in M_{n}(\mathbb{C})$ it is said that $A \stackrel{2,3}{\leq} B$ if $S_{A}^{2}(\lambda) \stackrel{\sharp}{\leq} S_{B}^{2}(\lambda)$ for all $\lambda \in \mathbb{C} \backslash\{0\}$ and $S_{A}^{3}(\lambda) \leq S_{B}^{3}(\lambda)$ for all $\lambda \in \mathbb{C}$.

It is clear that the cn-order coincides with the sharp partial order for matrices of index at most 1 and also coincides with the minus partial order for nilpotent matrices. This leads to the following lemmas.

Lemma 2.7. Let $A, B \in M_{n}(\mathbb{C})$. Then $A \stackrel{1}{\leq} B$ if and only if $S_{A}^{1}(\lambda) \stackrel{c n}{\leq} S_{B}^{1}(\lambda)$ for all $\lambda \in \mathbb{C}$. 
Proof. Follows directly from the definition of the cn-order and from the fact that $S_{M}^{1}(0)$ is nilpotent and $S_{M}^{1}(\lambda)$ is of index at most 1 for all $\lambda \in \mathbb{C} \backslash\{0\}$ and for any $M \in M_{n}(\mathbb{C})$. In particular, apply this reasoning to $M \in\{A, B\}$.

Lemma 2.8. Let $A, B \in M_{n}(\mathbb{C})$. Then $A \stackrel{2,3}{\leq} B$ if and only if $S_{A}^{2}(\lambda)=S_{B}^{2}(\lambda)$ for all $\lambda \in \mathbb{C} \backslash\{0\}$ and $S_{A}^{3}(\lambda) \leq S_{B}^{3}(\lambda)$ for all $\lambda \in \mathbb{C}$.

Proof. Since $S_{A}^{2}(\lambda)$ and $S_{B}^{2}(\lambda)$ are idempotent matrices, $S_{A}^{2}(\lambda) \stackrel{\sharp}{\leq} S_{B}^{2}(\lambda)$ is equivalent to $S_{A}^{2}(\lambda) \leq S_{B}^{2}(\lambda)$

Now, we can prove that these new binary relations are indeed partial orders.

Theorem 2.9. $\stackrel{1}{\leq}$ and $\stackrel{2,3}{\leq}$ are partial order relations on $M_{n}(\mathbb{C})$.

Proof. It is straightforward that $A \stackrel{1}{\leq} A$ and $A \stackrel{2,3}{\leq} A$ for all $A \in M_{n}(\mathbb{C})$.

Let $A \stackrel{1}{\leq} B$ and $B \stackrel{1}{\leq} A$. Then $S_{A}^{1}(\lambda)=S_{B}^{1}(\lambda)$ for all $\lambda \in \mathbb{C}$. Hence, $A=B$ by the first decomposition from Definition 2.3.

Let $A \stackrel{2,3}{\leq} B$ and $B \stackrel{2,3}{\leq} A$. Then $S_{A}^{3}(\lambda)=S_{B}^{3}(\lambda)$ for all $\lambda \in \mathbb{C}$ and $S_{A}^{2}(\lambda)=S_{B}^{2}(\lambda)$ for all $\lambda \in \mathbb{C} \backslash\{0\}$. However, $S_{M}^{2}(0)$ is included to the decomposition of $M$ given by Definition 2.3 with the 0 coefficient. Thus, again $A=B$.

Let $A \stackrel{1}{\leq} B \stackrel{1}{\leq} C$. Then for any $\lambda \in \mathbb{C}$ it holds that $S_{A}^{1}(\lambda) \stackrel{\text { cn }}{\leq} S_{B}^{1}(\lambda) \stackrel{\text { cn }}{\leq} S_{C}^{1}(\lambda)$. Hence, $S_{A}^{1}(\lambda) \stackrel{\text { cn }}{\leq} S_{C}^{1}(\lambda)$ since $\stackrel{\text { cn }}{\leq}$ is a partial order relation. Thus, by Lemma $2.7, A \stackrel{1}{\leq} C$.

Let $A \stackrel{2,3}{\leq} B \stackrel{2,3}{\leq} C$. Then for any $\lambda \in \mathbb{C}$ it holds that $S_{A}^{3}(\lambda) \stackrel{-}{\leq} S_{B}^{3}(\lambda) \stackrel{-}{\leq} S_{C}^{3}(\lambda)$ and for any $\lambda \in \mathbb{C} \backslash\{0\}$ it holds that $S_{A}^{2}(\lambda) \stackrel{\sharp}{\leq} S_{B}^{2}(\lambda) \stackrel{\sharp}{\leq} S_{C}^{2}(\lambda)$. Hence, $A \stackrel{2,3}{\leq} C$ by definition since $\leq$ and $\stackrel{\sharp}{\leq}$ are partial order relations.

It is well known that if $A \stackrel{\text { cn }}{\leq} B$ then $\operatorname{Spec} A \subseteq \operatorname{Spec} B \cup\{0\}$ (see [15, Theorem 4.4.18]). Similar relations are valid for $\stackrel{1}{\leq}$ - and $\stackrel{2,3}{\leq}$-orders.

Lemma 2.10. Let $A, B \in M_{n}(\mathbb{C})$ such that $A \stackrel{1}{\leq} B$ or $A \stackrel{2,3}{\leq} B$. Then $\operatorname{Spec} A \subseteq \operatorname{Spec} B \cup$ $\{0\}$. Moreover, if $0 \notin \mathrm{Spec} A$ or $A$ has a Jordan cell of size $k \geq 2$ with 0 eigenvalue, then $\operatorname{Spec} A \subseteq \operatorname{Spec} B$.

Proof. Let $\lambda \in \operatorname{Spec} A \backslash\{0\}$. If $A \stackrel{1}{\leq} B$ or $A \stackrel{2,3}{\leq} B$ then $S_{A}^{i}(\lambda) \stackrel{\sharp}{\leq} S_{B}^{i}(\lambda)$ for $i=1,2$, respectively. If we suppose $\lambda \notin \operatorname{Spec} B$ then $O \neq S_{A}^{i}(\lambda) \stackrel{\sharp}{\leq} S_{B}^{i}(\lambda)=O$, which is not possible. Hence, Spec $A \subseteq \operatorname{Spec} B \cup\{0\}$. In addition, if $A$ has a Jordan cell of size $k \geq 2$ with 0 eigenvalue then $S_{A}^{1}(0) \neq O$ and $S_{A}^{3}(0) \neq O$. Hence, considering the minus order, we have $S_{B}^{1}(0) \neq O$ and $S_{B}^{3}(0) \neq O$. Thus, $0 \in \operatorname{Spec} B$. 
The introduced orders are invariant under similarities.

Lemma 2.11. Let $A, B \in M_{n}(\mathbb{C})$. For all nonsingular $P \in M_{n}(\mathbb{C})$, it follows that

(a) $A \stackrel{1}{\leq} B$ implies $P A P^{-1} \stackrel{1}{\leq} P B P^{-1}$.

(b) $A \stackrel{2,3}{\leq} B$ implies $P A P^{-1} \stackrel{2,3}{\leq} P B P^{-1}$.

Proof. It is straightforward using item (iv) of Theorem 2.2 and the fact that minus, sharp and cn-orders are invariant under similarities.

\section{Relationships with other orders}

In this section we show that a matrix $A$ is below another matrix $B$ under the cn-order if and only if $A$ is below $B$ under the $\stackrel{1}{\leq}$ order. We will also prove that although this equivalence does not occur between the cn-order and the $\stackrel{2,3}{\leq}$-order, the implication

$$
A \stackrel{\mathrm{cn}}{\leq} B \quad \Longrightarrow \quad A \stackrel{2,3}{\leq} B
$$

remains valid.

We quote here the following result which is useful for our considerations.

Theorem 3.1. [15, Theorem 4.4.18] Let $A, B \in M_{n}(\mathbb{C})$. Then $A \stackrel{c n}{\leq} B$ if and only if there exist invertible matrices $P \in M_{n}(\mathbb{C}), C_{1} \in M_{k_{1}}(\mathbb{C}), C_{2} \in M_{k_{2}}(\mathbb{C})$ and nilpotent matrices $N_{1}, N_{2} \in M_{n-\left(k_{1}+k_{2}\right)}$, such that

$$
A=P\left(\begin{array}{ccc}
C_{1} & & \\
& O & \\
& & N_{1}
\end{array}\right) P^{-1} \quad \text { and } \quad B=P\left(\begin{array}{ccc}
C_{1} & & \\
& C_{2} & \\
& & N_{2}
\end{array}\right) P^{-1}
$$

with $N_{1} \leq N_{2}$

Now, we show that $\stackrel{1}{\leq}$-order follows from $\stackrel{\text { cn }}{\leq}$-order.

Lemma 3.2. Let $A, B \in M_{n}(\mathbb{C})$. If $A \stackrel{c n}{\leq} B$ then $A \stackrel{1}{\leq} B$.

Proof. Let $A \stackrel{\text { cn }}{\leq} B$. Clearly, the result is trivial for $A=O$. Hence, assume further that $A \neq O$, so $B \neq O$. Then by Theorem 3.1

$$
A=P\left(\begin{array}{ccc}
C_{1} & & \\
& O & \\
& & N_{1}
\end{array}\right) P^{-1} \quad \text { and } \quad B=P\left(\begin{array}{ccc}
C_{1} & & \\
& C_{2} & \\
& & N_{2}
\end{array}\right) P^{-1}
$$


with $N_{1} \leq N_{2}$ and $C_{1}, C_{2}$ invertible. Then from the uniqueness of the core-nilpotent decomposition of a matrix it follows that

$$
\begin{gathered}
C_{A}=P\left(\begin{array}{ccc}
C_{1} & & \\
& O & \\
& & O
\end{array}\right) P^{-1}, \quad N_{A}=P\left(\begin{array}{ccc}
O & & \\
& O & \\
& & N_{1}
\end{array}\right) P^{-1}, \\
C_{B}=P\left(\begin{array}{ccc}
C_{1} & & \\
& C_{2} & \\
& & O
\end{array}\right) P^{-1}, \quad \text { and } \quad N_{B}=P\left(\begin{array}{ccc}
O & & \\
& O & \\
& & N_{2}
\end{array}\right) P^{-1}
\end{gathered}
$$

are the core-nilpotent decompositions of $A$ and $B$, correspondingly, and we have $C_{A} \stackrel{\sharp}{\leq}$ $C_{B}$ and $N_{A} \leq N_{B}$.

In order to see that $A \stackrel{1}{\leq} B$ holds we have to demonstrate the inequalities $S_{A}^{1}(\lambda) \stackrel{\sharp}{\leq}$ $S_{B}^{1}(\lambda)$ for all $\lambda \in \mathbb{C} \backslash\{0\}$ and $S_{A}^{1}(0) \leq S_{B}^{1}(0)$. By definition, $S_{A}^{1}(0)=N_{A}$ and $S_{B}^{1}(0)=$ $N_{B}$, thus

$$
S_{A}^{1}(0) \stackrel{-}{\leq} S_{B}^{1}(0)
$$

is obtained directly.

Since similarities preserves the $\stackrel{\sharp}{\leq}$ order, it follows from $C_{A} \stackrel{\sharp}{\leq} C_{B}$ that

$$
\left(\begin{array}{ccc}
C_{1} & & \\
& O & \\
& & O
\end{array}\right) \stackrel{\sharp}{\leq}\left(\begin{array}{ccc}
C_{1} & & \\
& C_{2} & \\
& & O
\end{array}\right) .
$$

Let us compute the Jordan canonical forms of $C_{1}$ and $C_{2}$, that is, $C_{1}=S_{1} J_{1} S_{1}^{-1}$ and $C_{2}=S_{2} J_{2} S_{2}^{-1}$. Hence,

$$
\left(\begin{array}{ccc}
S_{1} J_{1} S_{1}^{-1} & & \\
& O & \\
& & O
\end{array}\right) \stackrel{\sharp}{\leq}\left(\begin{array}{ccc}
S_{1} J_{1} S_{1}^{-1} & \\
& S_{2} J_{2} S_{2}^{-1} & \\
& & O
\end{array}\right),
$$

which can be re-written as

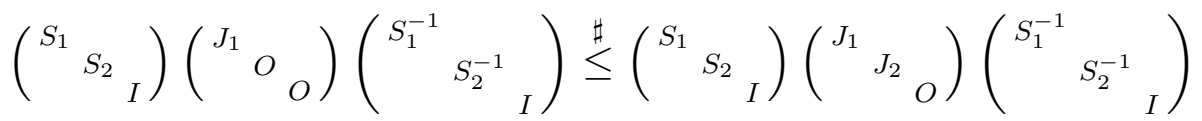

and is equivalent to

$$
A^{\prime}=\left(\begin{array}{ccc}
J_{1} & & \\
& O & \\
& & O
\end{array}\right) \stackrel{\sharp}{\leq}\left(\begin{array}{lll}
J_{1} & & \\
& J_{2} & \\
& & O
\end{array}\right)=B^{\prime} .
$$


We consider the diagonal concatenation of blocks with the same eigenvalue in the Jordan form:

$$
J_{1}=\left(\begin{array}{ccc}
A_{1} & \ldots & O \\
\vdots & \ddots & \vdots \\
O & \ldots & A_{p}
\end{array}\right), \quad J_{2}=\left(\begin{array}{cccccc}
B_{1} & \ldots & O & O & \ldots & O \\
\vdots & \ddots & \vdots & \vdots & \ddots & \vdots \\
O & \ldots & B_{p} & O & \ldots & O \\
O & \ldots & O & B_{p+1} & \ldots & O \\
\vdots & \ddots & \vdots & \vdots & \ddots & \vdots \\
O & \ldots & O & \ldots & \ldots & B_{q}
\end{array}\right)
$$

Here, for $i, j=1, \ldots, p$, we have $\operatorname{Spec} A_{i}=\left\{\lambda_{i}\right\}, 0 \notin \operatorname{Spec} A_{i}$, and $\operatorname{Spec} A_{i} \cap \operatorname{Spec} A_{j}=$ $\emptyset$ if $i \neq j$. Analogously, for $i=1, \ldots, p$, we have Spec $B_{i}=\left\{\lambda_{i}\right\}$ and moreover, for $i, j=1, \ldots, q, \operatorname{Spec} B_{i} \cap \operatorname{Spec} B_{j}=\emptyset$ if $i \neq j$ and for $k=p+1, \ldots, q, 0 \notin \operatorname{Spec} B_{k}$. We observe that some (or even all) of the blocks $B_{k}, k=p+1, \ldots, q$, may be absent. Also, the size of $B_{i}, i=1, \ldots, p$, and the one of $A_{i}$ may not be the same.

We get by (3.2) and (3.3) that
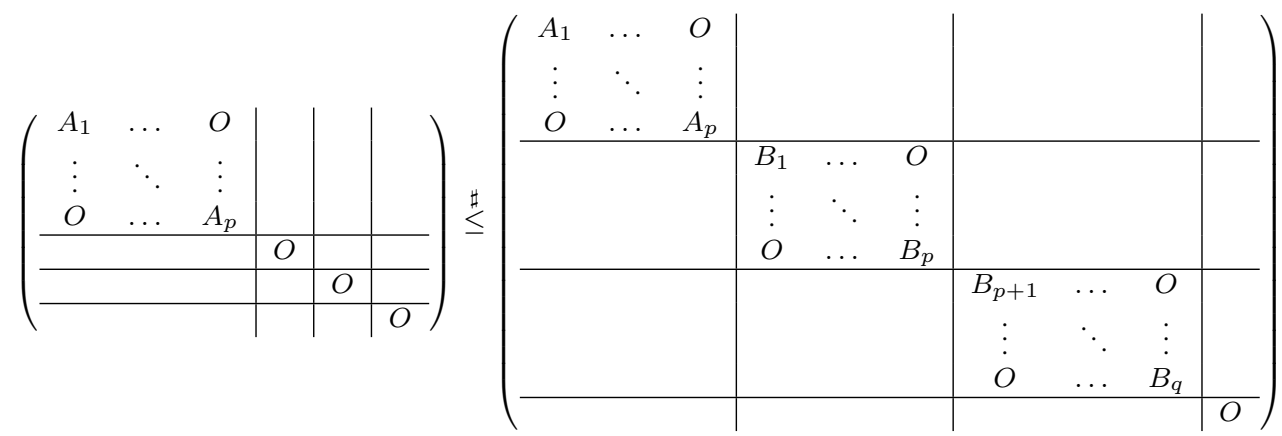

Note that, by definition, for each $i=1, \ldots, p$,

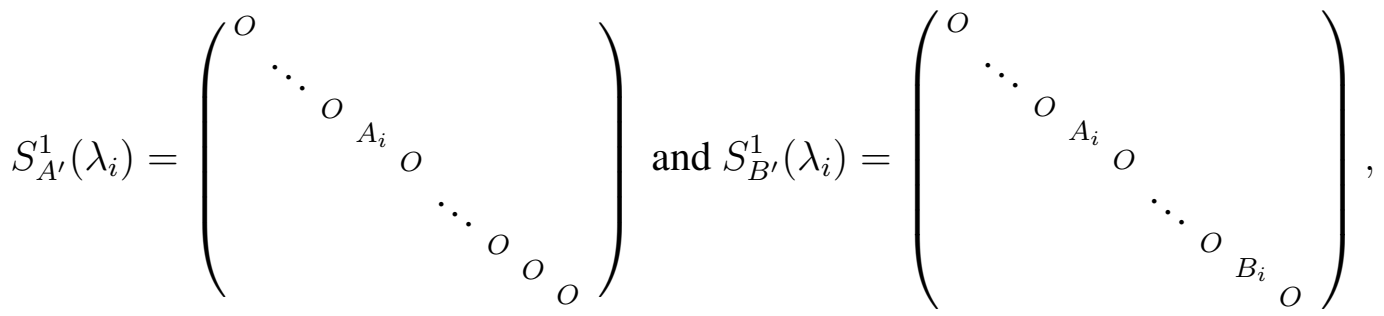

that is, in $S_{B^{\prime}}^{1}\left(\lambda_{i}\right)$ the block $A_{i}$ is located exactly at the same place as in $S_{A^{\prime}}^{1}\left(\lambda_{i}\right)$.

Then it is straightforward to check that for each $i=1, \ldots, p$ it holds that $S_{A^{\prime}}^{1}\left(\lambda_{i}\right) \stackrel{\sharp}{\leq}$ $S_{B^{\prime}}^{1}\left(\lambda_{i}\right)$. Therefore,

$$
S_{A}^{1}\left(\lambda_{i}\right) \stackrel{\sharp}{\leq} S_{B}^{1}\left(\lambda_{i}\right)
$$

since the $\stackrel{\sharp}{\leq}$-order is preserved under similarities (see item (iv) of Theorem 2.2).

For any $\lambda \in \mathbb{C} \backslash\left\{0, \lambda_{1}, \ldots, \lambda_{p}\right\}$,

$$
S_{A}^{1}(\lambda)=O \stackrel{\sharp}{\leq} S_{B}^{1}(\lambda) .
$$


Combining (3.1), (3.5), and (3.6), we get $A \stackrel{1}{\leq} B$.

The following lemma establishes that the cn-order also follows from the $\stackrel{1}{\leq}$-order.

Lemma 3.3. Let $A, B \in M_{n}(\mathbb{C})$. If $A \stackrel{1}{\leq} B$ then $A \stackrel{c n}{\leq} B$.

Proof. By Lemma 2.7, $A \stackrel{1}{\leq} B$ is equivalent to $S_{A}^{1}(\lambda) \stackrel{\text { cn }}{\leq} S_{B}^{1}(\lambda)$ for all $\lambda \in \mathbb{C}$. Hence, $S_{A}^{1}(0) \leq S_{B}^{1}(0)$, i.e. $N_{A} \leq N_{B}$.

We have to prove now that $S_{A}^{1}(\lambda) \stackrel{\sharp}{\leq} S_{B}^{1}(\lambda)$ for all $\lambda \in \mathbb{C} \backslash\{0\}$ implies $C_{A} \stackrel{\sharp}{\leq} C_{B}$, i.e.

$$
\sum_{\lambda \in \mathbb{C} \backslash\{0\}} S_{A}^{1}(\lambda) \stackrel{\#}{\leq} \sum_{\lambda \in \mathbb{C} \backslash\{0\}} S_{B}^{1}(\lambda) .
$$

It follows from the proof of Lemma 3.2 that for any $\lambda \neq 0$ and for any $X \in M_{n}(\mathbb{C})$, $S_{X}^{1}(\lambda)=S_{C_{X}}^{1}(\lambda)$. By definition, $S_{X}^{1}(\lambda) \stackrel{\sharp}{\leq} X$ for any $\lambda$. It then follows that $S_{A}^{1}(\lambda) \stackrel{\sharp}{\leq}$ $S_{B}^{1}(\lambda)=S_{C_{B}}^{1}(\lambda) \stackrel{\sharp}{\leq} C_{B}$ for any $\lambda \neq 0$, i.e., $S_{A}^{1}(\lambda) \stackrel{\sharp}{\leq} C_{B}$ for any $\lambda \neq 0$. Denote by $\lambda_{1}, \ldots, \lambda_{p}$ the set of nonzero eigenvalues of $A$. Since $S_{A}^{1}\left(\lambda_{i}\right) \perp S_{A}^{1}\left(\lambda_{j}\right)$ (see Theorem 2.2), it follows by [4, Theorem 2.22] that $S_{A}^{1}\left(\lambda_{1}\right)+S_{A}^{1}\left(\lambda_{2}\right) \stackrel{\sharp}{\leq} C_{B}$. In addition, $S_{A}^{1}\left(\lambda_{1}\right)+$ $S_{A}^{1}\left(\lambda_{2}\right) \perp S_{A}^{1}\left(\lambda_{3}\right)$. Hence, $\left(S_{A}^{1}\left(\lambda_{1}\right)+S_{A}^{1}\left(\lambda_{2}\right)\right)+S_{A}^{1}\left(\lambda_{3}\right) \stackrel{\sharp}{\leq} C_{B}$. Arguing in the same way we obtain that $\left(S_{A}^{1}\left(\lambda_{1}\right)+\ldots+S_{A}^{1}\left(\lambda_{p-1}\right)\right)+S_{A}^{1}\left(\lambda_{p}\right) \stackrel{\sharp}{\leq} C_{B}$. Using that $C_{A}=S_{A}^{1}\left(\lambda_{1}\right)+$ $\ldots+S_{A}^{1}\left(\lambda_{p}\right)$, we arrive at $C_{A} \stackrel{\sharp}{\leq} C_{B}$ and the result follows.

From Lemmas 3.2 and 3.3 we derive the following result which provides a characterization of the cn-order in terms of the spectrally orthogonal decomposition.

Theorem 3.4. Let $A, B \in M_{n}(\mathbb{C})$. Then $A \stackrel{1}{\leq} B$ if and only if $A \stackrel{c n}{\leq} B$.

In what follows, the relationship between the $\stackrel{\mathrm{cn}}{\leq}$ - and $\stackrel{2,3}{\leq}$-partial orders is analyzed.

Theorem 3.5. Let $A, B \in M_{n}(\mathbb{C})$. If $A \stackrel{c n}{\leq} B$ then $A \stackrel{2,3}{\leq} B$.

Proof. Suppose that $A \stackrel{\text { cn }}{\leq} B$. Clearly, $S_{A}^{3}(0)=S_{A}^{1}(0)=N_{A} \leq N_{B}=S_{B}^{1}(0)=S_{B}^{3}(0)$. Moreover, as we have obtained in the proof of Lemma 3.2, we can also get the expression (3.4). Then, for $i=1, \ldots, p$,

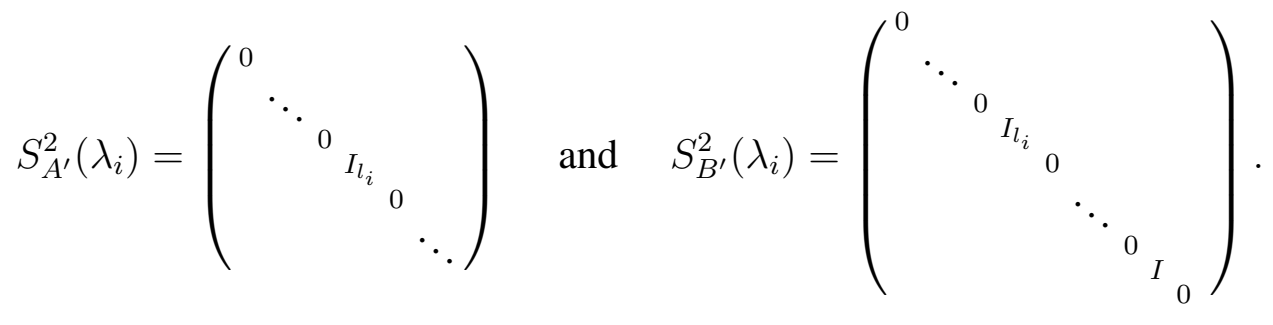


In both matrices, the block $I_{l_{i}}$ is located exactly at the same place, i.e., is a successor of the $l_{1}+\ldots+l_{i-1}$ square zero block. Hence, it is straightforward to see that $S_{A^{\prime}}^{2}\left(\lambda_{i}\right) \stackrel{\sharp}{\leq} S_{B^{\prime}}^{2}\left(\lambda_{i}\right)$.

Similarly,

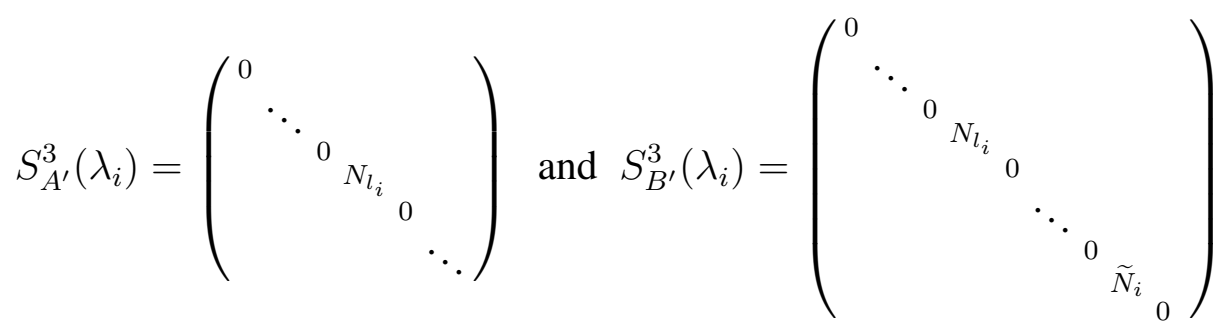

have both nonzero nilpotent blocks in the position of $A_{i}$ and, additionally, $S_{B^{\prime}}^{3}\left(\lambda_{i}\right)$ may have also some other nonzero diagonal block $\widetilde{N}_{i}$, which is not located on the position occupied by $A_{i}$. Hence, $S_{A^{\prime}}^{3}\left(\lambda_{i}\right) \stackrel{-}{\leq} S_{B^{\prime}}^{3}\left(\lambda_{i}\right)$.

Since minus and sharp partial orders are preserved under similarity transformations, $S_{A}^{2}\left(\lambda_{i}\right) \stackrel{\sharp}{\leq} S_{B}^{2}\left(\lambda_{i}\right)$ and $S_{A}^{3}\left(\lambda_{i}\right) \stackrel{-}{\leq} S_{B}^{3}\left(\lambda_{i}\right)$.

\subsection{Examples and counterexamples}

Below we provide a number of examples showing that the $\stackrel{2,3}{\leq}$-order introduced in this paper is essentially different from the extensions of the $\stackrel{\sharp}{\leq}$-order. In particular, it is different from the cn-order.

We start with an example showing that in general the $\stackrel{\text { cn }}{\leq}$-order does not follow from the $\stackrel{2,3}{\leq}$-order.

Example 3.6. Let $A \in M_{n}(\mathbb{C})$ be a Jordan cell with 1 on the main diagonal, i.e.

$$
A=J_{n}(1)=I+E_{12}+E_{23}+\ldots+E_{n-1, n} .
$$

Then it follows from Theorem 3.1, that $A$ is a minimal element of $M_{n}(\mathbb{C}) \backslash\{O\}$ with respect to the $\stackrel{\sharp}{\leq}$ - and $\stackrel{\mathrm{cn}}{\leq}$-orders. Indeed, a Jordan cell can not be similar to a block matrix of type $\left(\begin{array}{ccc}C_{1} & & \\ & C_{2} & \\ & & O_{k}\end{array}\right), k>0$, with invertible blocks $C_{1}$ and $C_{2}$. So, there is no nonzero $B \neq A$ such that $B \stackrel{\text { cn }}{\leq} A$. However $E_{i i} \stackrel{2,3}{\leq} A$ for all $i=1, \ldots, n$. Indeed, $S_{A}^{2}(\lambda)=S_{A}^{3}(\lambda)=O$ for all $\lambda \in \mathbb{C} \backslash\{1\}$,

$$
S_{A}^{2}(1)=I_{n}, \text { and } S_{A}^{3}(1)=J_{n}(0)=E_{12}+E_{23}+\ldots+E_{n-1, n} .
$$

For any $i, 1 \leq i \leq n$, we have $S_{E_{i i}}^{2}(1)=E_{i i}, S_{E_{i i}}^{2}(\lambda)=O$ for all $\lambda \in \mathbb{C} \backslash\{0,1\}$ and $S_{E_{i i}}^{3}(\lambda)=$ $O$ for all $\lambda \in \mathbb{C}$. Since $E_{i i} \stackrel{\sharp}{\leq} I_{n}$ and $O \leq S_{A}^{3}(\lambda)$ for all $\lambda \in \mathbb{C}$, it follows that $E_{i i} \stackrel{2,3}{\leq} A$ for all $i=1, \ldots, n$. 
Example 3.7. In the previous example it was shown that $E_{i i} \stackrel{2,3}{\leq} J_{n}(1)$. Observe that $E_{i i}$ is not below $J_{n}(1)$ with respect to the minus order. This shows that the $\stackrel{2,3}{\leq}$-order does not imply the minus order, in contrast with sharp and cn-orders.

Let us show that the $\stackrel{2,3}{\leq}$-order also does not follow from the minus order. We provide two different examples of matrices of different sizes in order to show that it is a general situation.

Example 3.8. 1) Consider the matrices

$$
A=\left(\begin{array}{ll}
0 & 1 \\
0 & 0
\end{array}\right) \quad \text { and } \quad B=\left(\begin{array}{cc}
0 & 1 \\
1 & 1
\end{array}\right)
$$

It is not hard to see that $A \overline{\leq} B$ but $A$ is not below $B$ under the $\stackrel{2,3}{\leq}$-order because $S_{A}^{3}(0)=$ $A$ is not below $S_{B}^{3}(0)=O$ under the minus order.

2) Similarly, the matrices

$$
A=\left(\begin{array}{llll}
0 & 1 & 0 & 0 \\
0 & 0 & 0 & 0 \\
0 & 0 & 0 & 0 \\
0 & 0 & 0 & 0
\end{array}\right) \quad \text { and } \quad B=\left(\begin{array}{rrrr}
0 & -1 & -2 & 0 \\
2 & 0 & -2 & 0 \\
0 & 0 & 0 & 0 \\
-2 & 2 & 4 & 0
\end{array}\right)
$$

satisfy that $A=B$ but $A$ is not below $B$ under the $\stackrel{2,3}{\leq}$-order because

$$
S_{A}^{3}(0)=A, \quad S_{B}^{3}(0)=-2 E_{43}
$$

and $\operatorname{rk}\left(S_{B}^{3}(0)-S_{A}^{3}(0)\right) \neq \operatorname{rk} S_{B}^{3}(0)-\operatorname{rk} S_{A}^{3}(0)$.

A well-known property for the minus, sharp and cn-orders is that if $A$ is strictly below $B$ with respect to one of these orders then $\operatorname{rk} A<\operatorname{rk} B$. The following example remarks that the $\stackrel{2,3}{\leq}$-order does not satisfy such a property.

Example 3.9. It is not hard to see that $I_{n} \stackrel{2,3}{\leq} J_{n}(1)$ but $\operatorname{rk} I_{n}=\operatorname{rk} J_{n}(1)=n$.

Remark 3.10. The above examples show that in contrast to all known extensions of the sharp order, see [15], the $\stackrel{2,3}{\leq}$-order is unrelated with the minus order and has nonstandard behavior with respect to the rank function.

Observe that some properties of the $\stackrel{2,3}{\leq}$-order introduced in this paper are just opposite to the properties of the sharp and cn-orders. 
Example 3.11. In Example 3.6, the matrix $A$ has been pointed out to be a minimal element of $M_{n}(\mathbb{C}) \backslash\{O\}$ with respect to the $\stackrel{\#}{\leq}$ - and $\stackrel{\text { cn }}{\leq}$-orders. Now, we establish that $A$ is a maximal element of $M_{n}(\mathbb{C})$ with respect to the $\stackrel{2,3}{\leq}$-order. Indeed, if there exists a matrix $B \in M_{n}(\mathbb{C})$ such that $A \stackrel{2,3}{\leq} B$ then $S_{B}^{2}(1)=I_{n}$ (that is, $\lambda=1$ is the only eigenvalue of $B)$ and $S_{B}^{2}(\lambda)=O$ for all $\lambda \neq 1$. Moreover, from $S_{A}^{3}(1)=A-I_{n} \leq S_{B}^{3}(1)$ and $S_{B}^{3}(1)$ is nilpotent we get rk $S_{B}^{3}(1)=n-1$. Hence, $S_{B}^{3}(1)=A-I_{n}$ since $\mathrm{rk}\left(A-I_{n}\right)=n-1$. Consequently, $B=S_{B}^{2}(1)+S_{B}^{3}(1)=A$.

\section{Some algebraic properties of the introduced order}

We start with the investigation of the behavior of the class of idempotent matrices under the considered orders.

Theorem 4.1. Let $A, B \in M_{n}(\mathbb{C})$ such that $A \stackrel{2,3}{\leq} B$. If $B$ is idempotent then $A$ is idempotent.

Proof. It is clear that $B^{2}=B$ assures the existence of a nonsingular $P \in M_{n}(\mathbb{C})$ such that $B=P(I \oplus O) P^{-1}$. By Lemma 2.10, we obtain that $\operatorname{Spec}(A) \subseteq \operatorname{Spec}(B) \cup\{0\} \subseteq\{0,1\}$. On the other hand, $A \stackrel{2,3}{\leq} B$ also implies $S_{A}^{2}(1) \stackrel{\sharp}{\leq} S_{B}^{2}(1)=B, S_{A}^{3}(1) \leq S_{B}^{3}(1)=O$ and $S_{A}^{3}(0) \leq S_{B}^{3}(0)=O$. Thus, $S_{A}^{3}(0)=O$ and $S_{A}^{3}(1)=O$. From items (v) and (vii) of Theorem 2.2, we arrive at $A=S_{A}^{2}(1)$, which is idempotent.

However, the converse of the previous theorem is not valid. Indeed, Example 3.6 shows that $E_{i i} \stackrel{2,3}{\leq} J_{n}(1)$ where $E_{i i}$ is idempotent and $J_{n}(1)$ is not.

We can say even more.

Theorem 4.2. Let $A, B \in M_{n}(\mathbb{C})$ be idempotent. The following conditions are equivalent:

(a) $A \stackrel{2,3}{\leq} B$

(b) $A \stackrel{c n}{\leq} B$.

(c) $A \stackrel{\sharp}{\leq} B$.

Proof. It is straightforward from [15, Theorem 4.2.8(iii)].

The most studied orders in the literature, namely minus, star, sharp, are $\mathcal{G}$-based. This means that $A \stackrel{\mathrm{G}}{\leq} B$ if and only if there exists a $g$-inverse $G \in M_{n}(\mathbb{C})$ of $A$ such that $A G=B G$ and $G A=G B$.

Note that, since the $\stackrel{1}{\leq}$-order is equivalent to the cn-order, it is not $\mathcal{G}$-based. We close this paper showing that, in general, the $\stackrel{2,3}{\leq}$-partial order is not $\mathcal{G}$-based on $M_{n}(\mathbb{C})$ as well. 
Remark 4.3. The order $\stackrel{2,3}{\leq}$ is not $\mathcal{G}$-based for any $n>1$. We consider the matrices

$$
A=\left(\begin{array}{cc}
1 & 0 \\
0 & 0
\end{array}\right) \oplus O_{n-2} \quad \text { and } \quad B=\left(\begin{array}{ll}
1 & 1 \\
0 & 1
\end{array}\right) \oplus O_{n-2}
$$

where $X \oplus Y$ denotes the block diagonal matrix with the blocks $X$ and $Y$ on the diagonal. It is straightforward to check that $A \stackrel{2,3}{\leq} B$. Suppose that there is a $g$-inverse $G \in M_{n}(\mathbb{C})$ of $A$ such that $A G=B G$ and $G A=G B$. Let $T=\frac{1}{2}(A+B)$. Then $T-A=\frac{1}{2}(B-A)$ and so, $A G=T G$ and $G A=G T$. Hence, $A \stackrel{2,3}{\leq} T$, which is a contradiction due to $S_{A}^{2}(1)$ is not below $S_{T}^{2}(1)$ under the sharp order.

Acknowledgments.

The authors thank the referees for their valuable comments. The first author is grateful to Mikhail A. Efimov for interesting discussions and to the Polytechnic University of Valencia for the warm hospitality and a partial financial support. Also his research was supported by the grants RFBR-15-01-01132 and MD-962.2014.1. The second and third authors have been partially supported by Ministerio de Economía y Competitividad from Spain, DGI MTM2013-43678-P.

\section{References}

[1] Baksalary, O.M., Trenkler, G.: Core inverse of matrices. Linear and Multilinear Algebra 58, 681-697 (2010)

[2] Baksalary, O.M., Trenkler, G.: On a generalized core inverse. Applied Mathematics and Computation 236, 450-457 (2014)

[3] Ben-Israel, A., Greville, T.: Generalized Inverses: Theory and Applications. Hohn Wiley and Sons, New York (1974)

[4] Guterman, A.E., Efimov, M.A.: Monotone maps on matrices of index one. Zap. Nauchn. Sem. POMI 405, 67-96 (2012) (in Russian); English transl.: Journal of Mathematical Sciences 405, 1-16 (2013)

[5] Guterman, A.E., Efimov, M.A.: Monotone maps on diagonalizable matrices, Mathematical Inequalities and Applications 17, 4, 1441-1452 (2014)

[6] Hartwig, R.E.: How to partially order regular elements. Math. Japonica 25, no. 1, $1-13$ (1980)

[7] Hartwig, R.E., Mitra, S.K.: Partial orders based on outer inverses. Linear Algebra Appl. 176, 3-20 (1982)

[8] Hernández A., Lattanzi M., Thome N., Urquiza F.: The star partial order and the eigenprojection at 0 on EP matrices, Applied Mathematics \& Computation, 218, 21, 10669-10678 (2012) 
[9] Hernández A., Lattanzi M., Thome N.: On a partial order defined by the weighted Moore-Penrose inverse, Applied Mathematics and Computation, 219, 14, 7310 7318 (2013)

[10] Hernández A., Lattanzi M., Thome N.: Weighted binary relations involving the Drazin inverse, Applied Mathematics and Computation, 253, 215-223 (2015)

[11] Lebtahi L., Patrício P., Thome N.: The diamond partial order in rings, Linear and Multilinear Algebra, 62, 3, 386-395 (2014)

[12] Malik S.B., Rueda L., Thome N.: Further properties on the core partial order and other matrix partial orders, Linear and Multilinear Algebra, 62, 12, 1629-1648, (2014)

[13] Meyer, C.D.: Matrix Analysis and Applied Linear Algebra. SIAM, Philadelphia (2000)

[14] Mitra, S.K.: On group inverses and the sharp order. Linear Algebra and its Applications, 92, 17-37 (1987)

[15] Mitra, S.K., Bhimasankaram, P., Malik, S.B.: Matrix Partial Orders, Shorted Operators and Applications. World Scientific Publishing Company (2010)

[16] Nambooripad, K.S.S.: The natural partial order on a regular semigroup. Proceedings of the Edinburgh Math. Soc. 23, 249-260 (1980)

[17] Rakić, D.S., Djordjević, D.S.: Space pre-order and minus partial order for operators on Banach spaces. Aequationes Mathematicae 85, 429-448 (2013) 